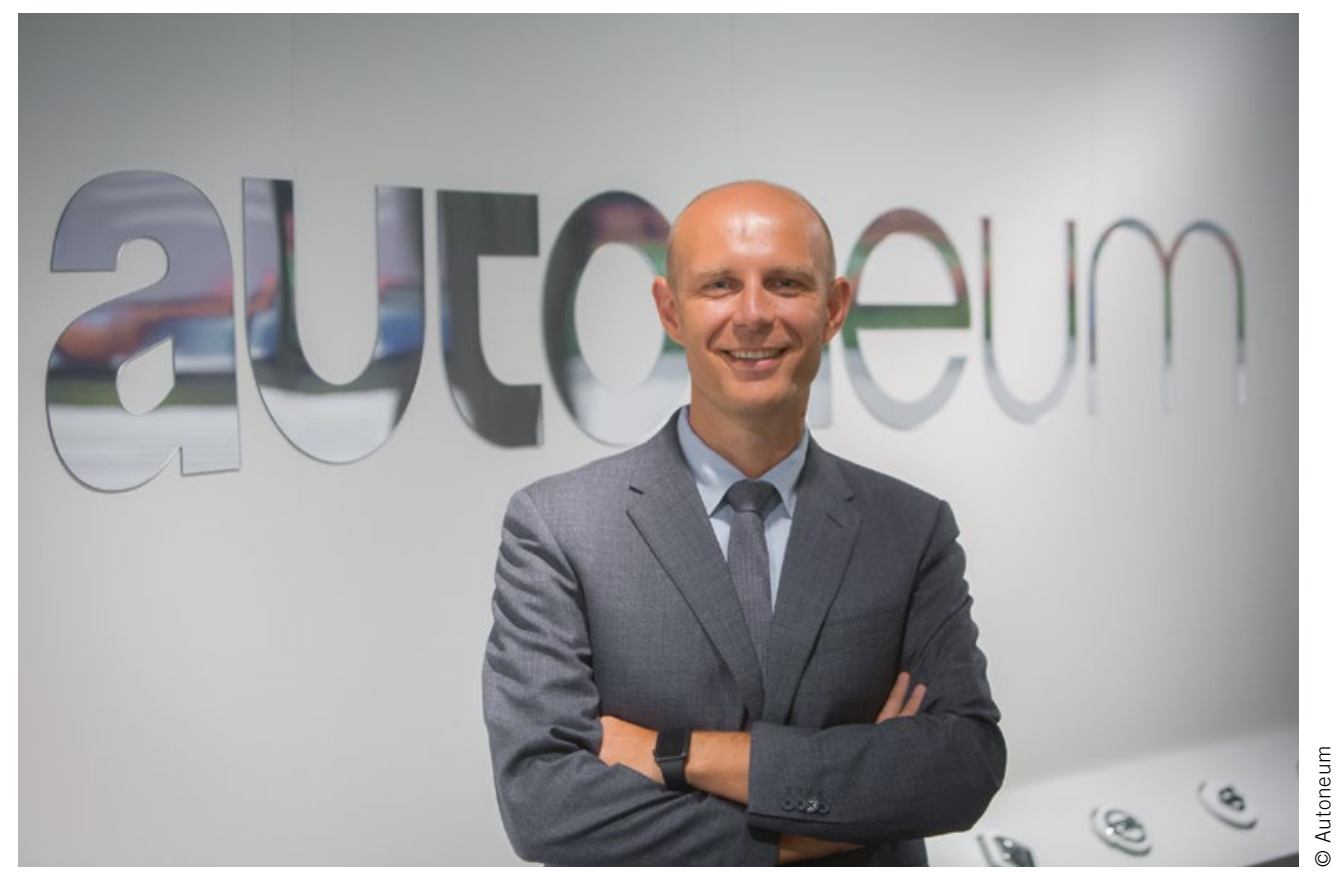

Dr. Davide Caprioli

Head of Product Acoustic and

Thermal Performance at Autoneum

\title{
The Role of NVH in Autonomous Driving
}

Autonomous driving is being seen as the next revolution in personal mobility. OEMs are increasingly thinking of themselves as mobility providers rather than simply vehicle manufacturers. Automotive companies such as Ford and Volvo are establishing alliances with Uber, Lyft and others. The aim of these alliances is to give the OEMs a competitive advantage. In addition, they are lobbying policymakers to create the regulatory framework needed to facilitate the use of self-driving vehicles.

What has this to do with NVH? Autonomous driving will change the way that drivers and passengers use the interior of a car. The requirements for passenger comfort will be even more demanding than they are today.

Because passengers will no longer need to pay attention to the traffic, they will become more sensitive to the acoustic environment. The increased communication within the passenger compartment and with remote contacts will need to be accompanied by a higher level of speech intelligibility. This might not be easy to achieve given that the new aesthetic requirements for the interior will involve the use of innovative materials for the door trims, headliner and carpet which have low or non-existent acoustic absorption properties.

Furthermore, the advent of hybrid and electric drive systems and the subsequent reduction in acoustic masking from the powertrain will highlight the need for further research by vehicle manufacturers and their suppliers into interior acoustic quality, including disturbances such as wind noise, road noise, the sounds made by ancillaries and squeak and rattle problems.
In addition, the moves towards more flexible seat configurations and the proliferation of safety, comfort and entertainment accessories in the passenger compartment will lead to increasing conflicts, which will result in greater geometric complexity and, in the worst case, a dramatic reduction in the package space available for interior NVH parts.

Over the last decade, we have already seen that the addition of the above-mentioned equipment has led to vehicles becoming larger and heavier. This in turn has a caused a growing demand for both weight and cost savings within the NVH package.

The next paradigm shift in the cost and weight breakdown of vehicles is just around the corner. We will soon be witnessing the extensive marketing of advanced autonomous driving assistance systems on a scale rarely seen in the automotive market. Goldman Sachs estimates that sales of smart sensors, radar and lidar last year amounted to 3 billion US dollars and that this figure will grow to 290 billion by 2035 .

Last but not least, autonomous driving will go hand in hand with new mobility concepts, such as car sharing, which will require greater wear resistance and easily replaced interior components.

As a consequence of these developments, engineers, material scientists, physicists and acoustics specialists need to think outside the box in order to develop innovations that can solve a seemingly impossible problem: providing enhanced passenger acoustic and ride comfort with solutions that are lighter in weight and lower in cost than those available today. 